\title{
Intoxicações na população infanto-juvenil atendidas em um centro de intoxicações do
}

\section{Nordeste Brasileiro}

Intoxications in the infanto-juvenile population cared for in an intoxication center in Northeast

Brazil

Intoxicaciones en la población infanto-juvenil atendida en un centro de intoxicación en el Noreste

de Brasil

Recebido: 20/05/2021 | Revisado: 28/05/2021 | Aceito: 31/05/2021 | Publicado: 15/06/2021

\author{
Emilianne Maria Silva Lopes \\ ORCID: https://orcid.org/0000-0002-7646-7681 \\ Universidade de Fortaleza, Brasil \\ E-mail: emilianne.lopes@hotmail.com \\ Igor Gomes de Araújo \\ ORCID: https://orcid.org/0000-0001-5427-8970 \\ Universidade de Fortaleza, Brasil \\ E-mail: gomes.igor1996@gmail.com \\ Erivan de Souza Oliveira \\ ORCID: https://orcid.org/0000-0002-0102-5475 \\ Universidade de Fortaleza, Brasil \\ E-mail: erivan@edu.unifor.br \\ Geysa Aguiar Romeu \\ ORCID: https://orcid.org/0000-0002-4899-1072 \\ Universidade de Fortaleza, Brasil \\ E-mail: geysa@unifor.br \\ Arlandia Cristina Lima Nobre de Morais \\ ORCID: https://orcid.org/0000-0002-5116-8546 \\ Universidade de Fortaleza, Brasil \\ E-mail: arlandia@unifor.br
}

\begin{abstract}
Resumo
O presente trabalho teve como objetivo analisar as intoxicações na população infanto-juvenil. Trata-se de um estudo descritivo, retrospectivo e com abordagem quantitativa, realizada em um hospital da rede pública terciário do Estado do Ceará. Foram avaliadas as fichas de intoxicações dos pacientes, suspeitos de intoxicações agudas por agentes tóxicos, no ano de 2019. Analisaram-se 959 fichas de pacientes com idade entre < 1 a 15 anos, e observado não haver diferença entre o sexo masculino $(50,89 \%, \mathrm{n}=488)$ e o feminino $(49,11 \%, \mathrm{n}=471)$. Em primeiro lugar, a faixa etária entre 1 a 5 anos, responsável por 43,80\% (n=420) dos casos, em segundo lugar, de 6 a 10 anos com 27,84\% $(\mathrm{n}=267)$, terceiro lugar de 11 a 15 anos com 26,80\% ( $\mathrm{n}=257)$, e em quarto e último < 1 ano de idade com 1,56\% $(\mathrm{n}=15)$. Em relação aos agentes tóxicos, escorpionismo foram os mais preeminentes, $78,62 \%(\mathrm{n}=754)$ dos casos, seguido por medicamentos com 6,05\% ( $\mathrm{n}=58)$, animais peçonhentos venenosos (serpentes) com 3,23\% ( $\mathrm{n}=31)$, animais não peçonhentos com 2,92\% $(\mathrm{n}=28)$, produtos químicos industriais 2,61\% $(\mathrm{n}=25)$ e os domissanitários com $1,25 \%$ $(\mathrm{n}=12)$. Com relação aos acidentes com animais peçonhentos, $80,65 \%(\mathrm{n}=25)$ dos casos foi causado por serpentes, $1,72 \%(n=13)$ por escorpião e 33,33\% $(n=2)$ por aranhas, necessitando em alguns casos o uso de antídotos. Conclui-se observar que os acidentes envolvendo as crianças e adolescentes estão se tornando cada vez mais frequentes, o que fomenta a necessidade de estratégias de campanhas para maiores orientações, acompanhamento e ajuda psicológica em escolas, espaços públicos e internet.
\end{abstract}

Palavras-chave: Intoxicação; Infanto-juvenil; Farmacêutico; Agentes tóxicos; Urgência e emergência.

\footnotetext{
Abstract

The present study aimed to analyze intoxications in children and adolescents. This is a descriptive, retrospective study with a quantitative approach, carried out in a public tertiary hospital in the State of Ceará. The intoxication records of patients, suspected of acute poisoning by toxic agents, were evaluated in 2019. 959 records of patients aged $<1$ to 15 years were analyzed, and it was observed that there was no difference between males $(50,89 \%, \mathrm{n}=488)$ and the female $(49.11 \%, n=471)$. First, the age group between 1 and 5 years, responsible for $43.80 \%(n=420)$ of the cases, in second place, from 6 to 10 years with $27.84 \%(\mathrm{n}=267)$, third place of 11 to 15 years old with $26.80 \%(\mathrm{n}=257)$, and in the fourth and last $<1$ year of age with $1.56 \%(\mathrm{n}=15)$. In relation to toxic agents, scorpionism were the most
} 
prominent, $78.62 \%(\mathrm{n}=754)$ of the cases, followed by drugs with $6.05 \%(\mathrm{n}=58)$, venomous venomous animals (snakes) with $3.23 \%(\mathrm{n}=31)$, non-venomous animals with $2.92 \%(\mathrm{n}=28)$, industrial chemicals $2.61 \%(\mathrm{n}=25)$ and household cleaning products with $1.25 \%(\mathrm{n}=12)$. With regard to accidents involving venomous animals, $80.65 \%(\mathrm{n}=$ $25)$ of the cases were caused by snakes, $1.72 \%(n=13)$ by scorpions and $33.33 \%(n=2)$ by spiders, requiring in some cases the use of antidotes. It is concluded that accidents involving children and adolescents are becoming more and more frequent, which promotes the need for campaign strategies for greater guidance, monitoring and psychological help in schools, public spaces and the internet.

Keywords: Intoxication; Children and youth; Pharmaceutical; Toxic agents; Urgency and emergency.

\section{Resumen}

El presente estudio tuvo como objetivo analizar las intoxicaciones en niños y adolescentes. Se trata de un estudio descriptivo, retrospectivo con abordaje cuantitativo, realizado en un hospital terciario público del Estado de Ceará. Los registros de intoxicación de pacientes, sospechosos de intoxicación aguda por tóxicos, fueron evaluados en 2019.Se analizaron 959 registros de pacientes <1 a 15 años, y se observó que no hubo diferencia entre varones (50, $89 \%, \mathrm{n}=488)$ y la mujer $(49,11 \%, \mathrm{n}=471)$. Primero, el grupo de edad entre 1 y 5 años, responsable del $43,80 \%(\mathrm{n}=$ 420) de los casos, en segundo lugar, de 6 a 10 años con 27,84\% ( $\mathrm{n}=267)$, tercer lugar de 11 a 15 años con 26,80\% (n $=257)$, y en el cuarto y último año $<1$ año con 1,56\% $(\mathrm{n}=15)$. En relación a los agentes tóxicos, el escorpionismo fue el más destacado, el 78,62\% $(\mathrm{n}=754)$ de los casos, seguido de los fármacos con el 6,05\% $(\mathrm{n}=58)$, los animales venenosos (serpientes) con el 3,23\% $(\mathrm{n}=31)$, animales no venenosos con $2,92 \%(\mathrm{n}=28)$, productos químicos industriales $2,61 \%(\mathrm{n}=25)$ y productos de limpieza del hogar con $1,25 \%(\mathrm{n}=12)$. En cuanto a los accidentes con animales venenosos, el 80,65\% $(\mathrm{n}=25)$ de los casos fueron causados por serpientes, el $1,72 \%(\mathrm{n}=13)$ por escorpiones y el 33,33\% ( $\mathrm{n}=2)$ por arañas, requiriendo en algunos casos el uso de antídotos. Se concluye que los accidentes que involucran a niños, niñas y adolescentes son cada vez más frecuentes, lo que promueve la necesidad de estrategias de campaña para una mayor orientación, seguimiento y ayuda psicológica en escuelas, espacios públicos e internet.

Palabras clave: Intoxicación; Infanto juvenil; Farmacéutico; Agentes tóxicos; Urgencia y emergencia.

\section{Introdução}

As intoxicações são apontadas como emergências médicas mais corriqueiras no público pediátrico, cujo agente doméstico predominante, são os domissanitários. O perigo da intoxicação em crianças se deve pelo pouco discernimento do que seja prejudicial à saúde e na maioria das vezes apresentam condutas curiosas (Aguiar, et al., 2020). Além disto, há uma ampliação nos casos envolvendo animais peçonhentos, sendo o escorpionismo com maior periodicidade nas regiões sudeste e nordeste do Brasil (Campos, et al., 2020).

A intoxicação exógena ou envenenamento pode ser caracterizada como um complexo de impactos nocivos determinado pelo surgimento de manifestações clínicas e/ou laboratoriais que resultam em uma desarmonia biológica, ocasionado pelo contato de uma ou mais fontes tóxicas com o organismo vivo (Almeida, et al., 2021; Oliveira, et al., 2021; Pereira, et al., 2021; Fernandes, et al., 2021).

Atualmente, no Brasil, sucedem-se cerca de 4,8 milhões de casos de intoxicações, onde, em torno de $0,1 \%$ a $0,4 \%$ findam-se em óbitos. Entre 1,5\% a 3\% da nação mundial é atingido por casos de intoxicação exógena (Alvim, et al., 2020; Melo, et al., 2021; Ferreira, et al., 2021; Soares, et al., 2021). Segundo informações obtidas no SINITOX (Sistema Nacional de Informações Tóxico-Farmacológicas) em 2017, ocorreram 76.115 intoxicações no Brasil, englobando pacientes com faixa etária desde menores de 1 ano até maiores de 80 anos de idade. Na faixa etária entre menores de 1 ano até 14 anos de idade, foram notificados 6.559 casos de intoxicações por medicamentos, 2.316 casos com domissanitários, 1.735 acidentes com escorpião, 1.375 acidentes com outros animais peçonhentos/venenosos e 1.178 casos de intoxicação com produtos químicos industriais (Sinitox, 2017).

Os envenenamentos acidentais são considerados como um transtorno de saúde universal. Diante do exposto, o presente trabalho teve como objeto analisar as intoxicações na população infanto-juvenil em um centro de intoxicação do Nordeste, determinando o perfil sociodemográfico, os agentes mais frequentes, avaliando a evolução dos pacientes e a conduta empregada. 


\section{Metodologia}

Trata-se de um estudo descritivo, retrospectivo e com abordagem quantitativa, realizada em um hospital da rede pública terciário do Estado do Ceará, pertencente a Secretaria Executiva Regional do Centro (SERCEFOR). A instituição é um centro de referência no Norte e Nordeste no socorro às vítimas de grandes traumas, queimaduras e envenenamento por animais, medicamentos e demais substâncias químicas.

Foram avaliadas as fichas de intoxicações dos pacientes atendidos no respectivo centro, suspeitos de intoxicações agudas por medicamentos, praguicidas, domissanitários, acidentes com animais peçonhentos (serpentes, aranhas e escorpiões), no ano de 2019.

A coleta de dados foi realizada utilizando formulário com as seguintes variáveis: iniciais do paciente, idade, sexo, procedência, mês do incidente, circunstâncias, agente intoxicante, manifestações clínicas, tratamento e evolução.

Os dados foram analisados por meio do programa Epi info, versão 7.2.2.6. As variáveis foram apresentadas como frequência absoluta (n) erelativa (\%).

A pesquisa foi aprovada pelo Comitê de Ética em Pesquisa com Seres Humanos do Hospital, onde a pesquisa ocorreu, sendo aprovado com parecer de $\mathrm{n}^{\circ} 3.550 .331$.

\section{Resultados}

Analisaram-se 959 fichas de pacientes com idade entre $<1$ a 15 anos, vítimas de intoxicações que foram atendidos presencialmente no setor de intoxicações, no ano de 2019. Foi observado que não houve diferença entre o gênero, acometendo tanto o sexo masculino $(50,89 \%, \mathrm{n}=488)$ como o sexo feminino $(49,11 \%, \mathrm{n}=471)$. Na classificação etária, verificou-se em primeiro lugar, a faixa etária entre 1 a 5 anos, responsável por 43,80\% $(n=420)$ dos casos, em segundo lugar, a faixa etária de 6 a 10 anos com 27,84\% ( $\mathrm{n}=267$ ), terceiro lugar a faixa etária de 11 a 15 anos com 26,80\% ( $\mathrm{n}=257)$, e em quarto e último a faixa etária $<1$ ano de idade com $1,56 \%(\mathrm{n}=15)$ (Tabela 1$)$.

Tabela 1 - Disposição das intoxicações conforme sexo e faixa etária. Ceará, Brasil, 2019.

\begin{tabular}{ccccccc}
\hline \multicolumn{7}{c}{ SEXO } \\
\hline FAIXA ETÁRIA & \multicolumn{2}{c}{ MASCULINO } & \multicolumn{2}{c}{ FEMININO } & \multicolumn{2}{c}{ TOTAL } \\
\hline \multicolumn{1}{c}{$\mathbf{N}$} & $\%$ & $\mathbf{N}$ & $\%$ & $\mathbf{N}$ & \% \\
\hline 1 a 5 anos & 8 & 53,33 & 7 & 44,67 & 15 & 1,56 \\
6 a 10 anos & 233 & 55,48 & 187 & 44,52 & 420 & 43,80 \\
11 a 15 anos & 127 & 47,57 & 140 & 52,43 & 267 & 27,84 \\
\hline TOTAL & 120 & 46,69 & 137 & 53,31 & 257 & 26,80 \\
\hline
\end{tabular}

Fonte: Autores (2020).

Em relação aos agentes tóxicos, notou-se que casos de escorpionismo foram os mais preeminentes, contabilizando $78,62 \%(n=754)$ dos casos, seguido por medicamentos com 6,05\% $(n=58)$, animais peçonhentos venenosos (serpentes) com $3,23 \%(\mathrm{n}=31)$, animais não peçonhentos com 2,92\% ( $\mathrm{n}=28)$, produtos químicos industriais 2,61\% $(\mathrm{n}=25)$ e os domissanitários com $1,25 \%(\mathrm{n}=12)$. De acordo com a exposição, mordedura/picada de animais obteve $88,11 \%(\mathrm{n}=845)$ dos acontecimentos, 
corroborando com os principais agentes tóxicos envolvidos (Tabela 2). Ressalta-se que todos as situações foram consideradas intoxicações agudas com exposição única. Cerca de 93,43\% (n=896) dos pacientes apresentou alguma manifestação clínica decorrente da intoxicação, 5,01\% $(n=48)$ dos pacientes necessitaram de internação hospitalar, entretanto 99,27\% (952) evoluíram para cura confirmada.

Tabela 2 - Perfil das intoxicações conforme o agente tóxico. Ceará, Brasil, 2019.

\begin{tabular}{ccc}
\hline AGENTE TÓXICO & \% & N \\
\hline Escorpionismo & $78,62 \%$ & 754 \\
Medicamentos & $6,05 \%$ & 58 \\
Outros animais peç.venenosos & $3,23 \%$ & 31 \\
Animais peç. serpentes & $3,23 \%$ & 31 \\
Animais não peçonhentos & $2,92 \%$ & 28 \\
Produtos químicos industriais & $2,61 \%$ & 25 \\
Domissanitários & $1,25 \%$ & 12 \\
Animais peç. aranhas & $0,63 \%$ & 6 \\
Agrotóxicos/agrícolas & $0,10 \%$ & 1 \\
Agrotóxico/ doméstico & $0,73 \%$ & 7 \\
Produtos veterinários & $0,31 \%$ & 3 \\
Raticidas & $0,63 \%$ & 6 \\
Alimentos & $0,10 \%$ & 1 \\
Cosméticos & $0,21 \%$ & 2 \\
\hline
\end{tabular}

Fonte: Autores (2020).

Com relação aos acidentes com animais peçonhentos, 80,65\% $(\mathrm{n}=25)$ dos casos foi causado por serpentes, $1,72 \%$ $(n=13)$ por escorpião e 33,33\% $(n=2)$ por aranhas, necessitando em alguns casos o uso de antídotos. Conforme a gravidade das intoxicações, averiguou-se que 93,22\% (n=894) foram diagnosticadas com envenenamento leve, 3,02\% (n=29) com envenenamento moderado, $073 \%(\mathrm{n}=7)$ envenenamento grave, 2,82\% (n=27) não intoxicação e 0,21\% (n=2) provável não tóxico.

Quanto a procedência dos pacientes atendidos observou-se que 89,67\% ( $\mathrm{n}=860)$ são provenientes da capital, logo, 10,33\% (n=99) são oriundos dos diversos interiores do Estado. Quanto as circunstâncias que as intoxicações ocorreram, o mais prevalente foram os acidentes individuais com 97,29\% (n=933) dos casos, seguido pela tentativa de suicídio com 2,29\% ( $n=22)$, automedicação com $0,21 \%(n=2)$, uso terapêutico com $0,10 \%(n=1)$ e ignorado com $0,10 \%(n=1)$. É de extrema importância ressaltar sobre as tentativas de autoextermínio, pois, foi notável que dos 58 casos de intoxicação medicamentosa, $55,17 \%(n=32)$ estão relacionados a intoxicação acidental e 37,93\% $(n=22)$ à tentativa de suicídio. Esta conduta ocorreu na faixa etária entre 11 a 15 anos com 7,78\% (n=20) dos casos, seguido da faixa etária de 1 a 5 anos e 6 a 10 anos, com um caso cada. Dos 7 envenenamentos graves, 71,43\% (n=5) estão relacionados a tentativa de autoextermínio e 85,71\% (n=6) estão relacionados a intoxicação medicamentosa e necessitaram de internação hospitalar.

\section{Discussão}

Devido à alta constância e morbilidade, os envenenamentos são classificados como um transtorno de saúde pública em toda a população mundial. Vários trabalhos têm reportado que crianças entre 1 a 5 anos são alvos mais prevalentes de 
intoxicações por diversos fatores intrínsecos que contribuem para o acontecimento desse problema, entre eles pode-se citar: o desconhecimento; o ambiente em que ela está inserida; as substâncias que estão no mesmo âmbito de convivência, como exemplo a presença de domissanitários, medicamentos armazenados em locais ao alcance, ou posologia inadequada na administração domiciliar pelos pais, ocasionando intoxicação exógena aguda (Amorim, et al., 2017; Silva, et al., 2019). Assim, como o tamanho pequeno de cápsulas e comprimidos, líquidos coloridos, cheiro agradável, cores exuberantes, o fácil acesso, a inocência e até mesmo a curiosidade espontânea da criança são condições que cooperam para este quadro. Soma-se a isso a falta de conhecimento ou a desatenção dos pais e responsáveis que contribuem significativamente para esse evento (Bochner, 2005).

Nesse estudo a faixa etária prevalente dos casos intoxicações foram entre 1 a 5 anos, vale salientar que nessa faixa o organismo ainda é imaturo, por isso em alguns casos pode ser mais letal (Oliveira, et al., 2014). Os resultados obtidos nessa pesquisa corroboram com o estudo de Maior, et al., (2020), que reportam que cerca de 7,3\% dos casos de intoxicações por medicamentos em crianças menores de 5 anos necessitaram de internação.

No estudo realizado por Vilaça \& Cardoso (2014), verificou-se mais de 140 mil atendimentos hospitalares de pacientes intoxicados, onde 6 mil foram a óbito, sendo as mais afetadas crianças com até 14 anos, ratificando que trata-se de uma questão de saúde pública. Ressalta-se que a adolescência é uma fase que se passa por diversas modificações, sendo elas de ordens hormonais, psíquicas e/ou sociais. A puberdade é um estágio de descobrimento de emoções e experiências até então inéditas para os jovens. Em virtude, é a etapa onde o perigo mostra-se aumentado para o uso de substâncias nocivas à saúde (Bochner, 2005).

Silva et al. (2019) reporta que durante o período de 2013 a 2017 cerca de $62,79 \%$ dos atendimentos de pacientes intoxicados eram do sexo feminino, diferente do encontrado no presente estudo que teve predomínio do sexo masculino com $50,89 \%$ dos casos.

No tocante aos agentes tóxicos, constatou que escorpionismo ficou no topo no número de casos, possuindo maior ocorrência na faixa etária de 1 a 5 anos. As ocorrências decorrentes de escorpionismo ocorreram por todo o território brasileiro com maior preponderância no nordeste e sudeste, pois, demonstrou-se que em 2017 houve 125.156 notificações, das quais 14 pessoas foram a óbito (Campos, et al., 2020). Esse resultado pode ser explicado pela ocorrência do crescimento das áreas urbanas, principalmente pelo desmatamento, além do uso de agrotóxicos que favorecem a mudança de habitat dos animais peçonhentos (Domingos, et al., 2016).

Uma vertente dentro das intoxicações que necessita de grande atenção é a tentativa de suicídio. Este trabalho apresentou informações que foram contabilizados como tentativa de autoextermínio, com predominância na faixa etária de 11 a 15 anos. As circunstâncias que levam ao jovem nessa idade a realizar o autoextermínio é consequência de diversas modificações e pode estar ligada a alguma doença psíquica como depressão e esquizofrenia, além de relações hostis familiares, disparidade de gênero e agressões familiares (Alvim, et al., 2020). É a forma de desafogar os pensamentos ruins, o abandono, o desespero, as mágoas, o esgotamento psicológico, a ansiedade, ou seja, o suicídio torna-se uma forma de lidar com as frustrações. A puberdade é um estágio de descobrimento de emoções e experiências até então inéditas para os jovens. É nesse período que o perigo se mostra aumentado para o uso de substâncias nocivas à saúde (Bochner, 2005; Cachão, et al., 2017).

Em comparação com o estudo de Sousa et al., (2020) realizado no Estado do Piauí, do Nordeste Brasileiro, os casos de intoxicações por medicamentos analisadas nos anos de 2007 a 2017, foi na grande maioria por tentativas de suicídio com 52,99\% ( $\mathrm{n}=2.132$ ) dos casos (Sousa, et al., 2020). Por ser julgado um prejuízo evitável, cabe ao Governo veicular campanhas de esclarecimento à população visando a orientação familiar, habilitar profissionais de saúde e propor ajuda psicológica em centros especializados aos casos de pessoas que sobressaíram da tentativa de suicídio, além de elaborar conteúdo socioeducativo para distribuir em escolas e universidades (Domingos, et al., 2016). 
Em conformidade com o fechamento deste estudo, reforça a frágil relação as medidas de precauções. Segundo Tavares et al. (2013), acentuar campanhas em saúde ainda na infância mostra-se efetivo na atenuação dos casos de intoxicação.

É notório que houve o aumento no número de casos de intoxicações, tanto com relação a escorpionismo, como medicamentos e domissanitários, o que leva a uma maior atenção, pois são agentes bastantes perigosos a vida e saúde das pessoas, em especial as crianças. Ressalta-se veementemente a importância do profissional farmacêutico frente aos casos de intoxicação, sendo um profissional habilitado e ponderado a atuar perante essas circunstâncias. É importante que o Governo adote medidas mais rigorosas com relação as embalagens de produtos domissanitários a fim de prevenir o armazenamento domiciliar inadequado de produtos químicos e que as crianças não tenham contato com essas substâncias.

\section{Considerações Finais}

É possível observar que os acidentes envolvendo as crianças e adolescentes estão se tornando cada vez mais frequentes, o que fomenta a necessidade constante de estratégias de campanhas para maiores orientações, acompanhamento e ajuda psicológica em escolas, espaços públicos e internet.

Por ser considerado um malefício refutável, os casos de envenenamento necessitam rigorosamente de atenção tanto pelos profissionais, cidadãos e o governo. Para que haja maior incentivo em educação em saúde, faz-se necessário investimentos governamentais em campanhas educativas, nos centros de atendimentos toxicológicos, espaços educacionais e principalmente avaliação de possíveis riscos que possam surgir, ratificando o fato de que as intoxicações são prejuízos evitáveis.

Indica-se também a necessidade da atualização dos bancos de dados como o SINITOX, pois, ajudaria significativamente o acompanhamento das evoluções e estudo das intoxicações. Recomenda-se a realização de mais estudos que tenham o foco de traçar os resultados encontrados no referido Estado, com os demais.

\section{Referências}

Aguiar, K. V. C. S., Cruz, R. C., Silva, R. T. A., \& Bonfim, A. S. (2020). Intoxicação exógenaacidental em crianças no estado da Bahia: 2013 a 2017. REAS, 12 (11), 3422. https://acervomais.com.br/index.php/saude/article/view/3422

Almeida, A. K. M., Oliveira, E. S., Araújo, I. G., Monteiro, F. F. C., Albuquerque, P. L. M. M., Romeu, G. A., \& Morais, A. C. L. N. (2021). Intoxicações por domissanitários notificados no período de 2015 a 2019 no Estado do Ceará. Revista de Casos e Consultoria, 12 (1), 23758. https://periodicos.ufrn.br/casoseconsultoria/article/view/23758

Alvim, A. L. S., França, R. O., Assis, B. B., \& Tavares, M. L. O. (2020). Epidemiologia da intoxicação exógena no Brasil entre 2007 e 2017. J. of Develop, 31 (8), 63915-63925.

Amorim, M. L. P., Mello, M. J. G., \& Siqueira, M. T. (2017). Intoxicações em crianças e adolescentes notificados em um centro de toxicologia no nordeste do Brasil. Rev.Bras. Saúde Mater. Infant., 17 (4), 765-772. https://www.scielo.br/scielo.php?script=sci_arttext\&pid=S1519-38292017000400765\&lng=en

Bochner, R. (2005). Papel da Vigilância Sanitária na prevenção de intoxicações na infância. REVISA: Revista Brasileira de Vigilância Sanitária, 1 (1), 50-57. https://www.arca.fiocruz.br/handle/icict/464

Cachão, J., Oliveira, I., \& Raminhos, I. (2017). Adolescência e Abuso de Substâncias. Nascer e Crescer, 26 (2), 103-108. http://www.scielo.mec.pt/scielo.php?script=sci_arttext\&pid=S0872-07542017000200004\&lng=en

Campos, L. L., Cardoso, F. L., \& Filho, A. A. (2020). Fisiopatologia e tratamento dos efeitos cardiovasculares e pulmonares no envenenamento por escorpião. Revista Médica de Minas Gerais, 30, 30203. http://www.rmmg.org/artigo/detalhes/2679

Domingos, S. M., Borghesan, N. B. A., Merino, M. F. G. L., \& Higarashi, I. H. (2016). Internações por intoxicação de crianças de zero a 14 anos em hospital de ensino no Sul do Brasil, 2006- 2011. Epidemiol. Serv. Saúde, 25 (2), 343-350. https://www.scielo.br/scielo.php?pid=S2237$96222016000200343 \&$ script=sci_abstract\&tlng=pt

Fernandes, A. R. C., Moura, G. P., \& Oliveira, G. L. (2021) Intoxicação por agrotóxico: ausência de registros no Sistema de Saúde do Estado da Bahia. Brazilian Journal of Development, 7 (5), 44574-44586. https://www.brazilianjournals.com/index.php/BRJD/article/view/29289

Ferreira, J. S., Silva, G. N. S., Beserra, B. A. S., Alves, S. R. P., \& Ferreira, A. F. (2021). Perfil epidemiológico das pessoas atendidas por intoxicação exógena em uma Unidade de Pronto Atendimento. Revista de Ciências da Saúde Nova Esperança, 19 (1), 06-12. http://revistanovaesperanca.com.br/index.php/revistane/article/view/658 
Research, Society and Development, v. 10, n. 7, e12210716329, 2021

(CC BY 4.0) | ISSN 2525-3409 | DOI: http://dx.doi.org/10.33448/rsd-v10i7.16329

Melo, A. B. B., Bueno, M. S., \& Silvério, A. P. (2021). Aspectos epidemiológicos dos casos notificados de intoxicação exógena por medicamentos no Estado do Tocantins entre 2016 e 2019. Facit Business and Technology Journal, 1 (25), 228-237. https://jnt1.websiteseguro.com/index.php/JNT/article/view/921

Oliveira, E. S., Liberato, F. L. R., Romeu, G. A., \& Morais, A. C. L. N. (2021). Intoxicação por antidepressivo tricíclico (amitriptilina): relato de caso. Revista de Casos e Consultoria, 12 (1), 24599. https://periodicos.ufrn.br/casoseconsultoria/article/view/24599

Oliveira, F. F. S., \& Suchara, E. A. (2014). Perfil epidemiológico das intoxicações exógenas em crianças e adolescentes em município do Mato Grosso. Rev. Paul. Pediatr, 32 (4), 299-305. https://www.scielo.br/scielo.php?pid=S0103-05822014000400299\&script=sci_abstract\&tlng=pt

Pereira, M. J. A., Pereira, A. J. A., Oliveira, D. R., Nicácio, B. S., Sá, J. C. L., Coelho, J. L. G., Carneiro, A. P. L., \& Gadelha, M. S. V. (2021). Perfil dos casos notificados de intoxicação exógena por medicamentos no Estado do Ceará. Revista Multidisciplinar e de Psicologia, 15 (54), 457-477. https://idonline.emnuvens.com.br/id/article/view/2984

Silva, M. A., Jesus, L. L. S., \& Branco, A. C. S. (2019). Intoxicações medicamentosas: um estudo através de dados secundários no Brasil. Research, Society and Development, 9 (2), 200922254. https://www.rsdjournal.org/index.php/rsd/article/view/2254/1843

Sinitox. (2017). Sistema Nacional de Informações Tóxico-Farmacológicas. https://sinitox.icict.fiocruz.br/sites/sinitox.icict.fiocruz.br/files//Brasil7_1.pdf

Soares, J. Y. S., Lima, B. M., Verri, I. A., \& Oliveira, S. V. (2021). Perfil epidemiológico de intoxicação exógena por medicamentos em Brasília. Revista de Atenção à Saúde, 19 (67), 202-217. https://www.seer.uscs.edu.br/index.php/revista_ciencias_saude/article/view/7335

Sousa, E. S. F., Carvalho, F. S., Macêdo, K. P. C., Leal, B. S., Feitosa, C. L. P., Rodrigues, M. M. M., Lourenço, S. S., Silva, R. S. V., Santos, M. C., Sousa, P. A., Lima, G. I. S., Arrais, K. W. N., Sá, N. K. C. M., Oliveira, E. H., \& Lúcio Neto, M. P. (2020). Análise das intoxicações por medicamentos no Piauí entre os anos de 2007 a 2017. REAS, 51, 745 .

Tavares, E. O., Buriola, A. A., Santos, J. A. T., Ballani, T. S. L., \& Oliveira, M. L. F. (2013). Fatores associados à intoxicação infantil. Esc. Anna Nery, 17 (1), 31-37. https://www.scielo.br/scielo.php?script=sci_arttext\&pid=S1414-81452013000100005

Vilaça, L., \& Cardoso, R. P. (2014). Intoxicações na infância: panorama geral do perfil das intoxicações em diferentes países. Rev Med Minas Gerais, 24 (1), 21-25. http://rmmg.org/artigo/detalhes/597 